Controlling non-radiative energy transfer in organic binary blends: a route towards colour tunability and white emission from single-active-layer light-emitting devices

This content has been downloaded from IOPscience. Please scroll down to see the full text. 2003 J. Phys. D: Appl. Phys. 362483

(http://iopscience.iop.org/0022-3727/36/20/009)

View the table of contents for this issue, or go to the journal homepage for more

Download details:

IP Address: 134.121.47.100

This content was downloaded on 02/10/2015 at 02:39

Please note that terms and conditions apply. 


\title{
Controlling non-radiative energy transfer in organic binary blends: a route towards colour tunability and white emission from single-active-layer light-emitting devices
}

\author{
Dario Pisignano ${ }^{1,2,4}$, Marco Mazzeo ${ }^{1,2}$, Giuseppe Gigli ${ }^{1}$, \\ Giovanna Barbarella ${ }^{3}$, Laura Favaretto ${ }^{3}$ and Roberto Cingolani ${ }^{1}$ \\ ${ }^{1}$ NNL, National Nanotechnology Laboratory of Istituto Nazionale di Fisica della \\ Materia (INFM), c/o Dipartimento di Ingegneria dell'Innovazione, via Arnesano, I-73100 \\ Lecce, Italy \\ ${ }^{2}$ Dipartimento di Fisica, Università di Lecce, via Arnesano, I-73100 Lecce, Italy \\ ${ }^{3}$ Consiglio Nazionale delle Ricerche (CNR), ICOCEA, Area della Ricerca di Bologna, via \\ Gobetti 101, I-40129 Bologna, Italy
}

E-mail: dario.pisignano@unile.it

Received 10 July 2003

Published 1 October 2003

Online at stacks.iop.org/JPhysD/36/2483

\begin{abstract}
We show how colour tunability (including white) can be achieved by controlling non-radiative intermolecular energy transfer from the donor to the acceptor in binary blends of oligomeric compounds. Blends of different concentrations of a novel functionalized thiophene-based oligomer and a low-molar-mass diamine derivative $\left(N, N^{\prime}\right.$-diphenyl- $N, N^{\prime}$-bis(3-methylphenyl)-1, $1^{\prime}$-biphenyl-4.4'diamine) are used to tune both the photoluminescence and the electroluminescence (EL) from red to blue, including balanced white, according to the standards of the Commission Internationale de l'Eclairage. The single-active-layer light-emitting devices, realized by spin-coating, exhibit good EL performance. In particular, the white-emitting device shows an EL efficiency of $5 \times 10^{-1} \mathrm{~cd} \mathrm{~A}^{-1}$ and a luminance of more than $180 \mathrm{~cd} \mathrm{~m}^{-2}$.
\end{abstract}

In the past few years organic-based light-emitting diodes (OLEDs) have been the subject of increasing interest due to their good electroluminescence (EL) performance (low turn-on voltage, high efficiency and high luminance), extreme flexibility and cheap fabrication technology. In particular, the achievement of both wide colour tunability (from blue to red) and white emission could have a tremendous impact on the lighting industry and backlight applications. To date, the most common approach to realizing white OLEDs is based on combining the emission from materials of different HOMOLUMO gaps, $E_{\mathrm{g}}$, in multilayer evaporated structures [1-3]. Using a unique blend including both the low- and high-energy emitters, by a one-step spin-coating fabrication procedure, would be undoubtedly advantageous in terms of cost and throughput. Nevertheless, Förster energy transfer would result

4 Author to whom any correspondence should be addressed. in emission from the smaller band gap material, provided that a sufficient overlap between the absorption spectrum of the acceptor and the emission spectrum of the donor occurs [4]. For this reason, an insulating and optically inert matrix was employed in previous studies to embed polymer blends, so reducing the rate of exciton transfer $[5,6]$. Other approaches to obtaining white emission include dispersion of dyes in an active polymeric environment [7,8], polymer blends [6], and formation of an exciplex between two blended blueemitting organic molecules [9]. Values of luminance up to $50000 \mathrm{~cd} \mathrm{~m}^{-2}$ and EL efficiency up to $3 \%$ from evaporated multilayer OLEDs have been reported [10]

In this paper, we show that the intermolecular energy transfer between oligomer molecules can be finely controlled by varying the relative concentration between the donor and the acceptor. This allows us to employ a unique binary blend of low-molar-mass molecules to achieve colour tunability, from 
red to blue, including balanced white, in single-active-layer OLEDs. We propose that this mechanism can be exploited as a general route to tune the emission from binary oligomer blends, resulting in a remarkable advantage for fabrication of single-active-layer multicolour OLEDs.

Our OLEDs were prepared by spin-coating, onto preliminary $\mathrm{O}_{2}$-plasma treated indium-tin-oxide (ITO) coated glass substrates, a hole-transporting layer, namely poly $(3,4-$ ethylene dioxythiophene) (PEDOT) doped with poly(styrene sulfonate) (PSS). Subsequently, we spin-cast the active medium, composed of a blend of $N, N^{\prime}$-diphenyl- $N, N^{\prime}$ bis(3-methylphenyl)-1, 1'-biphenyl-4.4' diamine (TPD) and of the functionalized thiophene-based oligomer, 3, 3', $4^{\prime \prime \prime}, 3^{\prime \prime \prime \prime}$ -

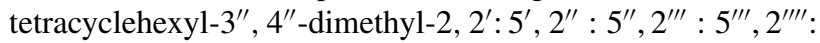
quinquethiophene- $1^{\prime \prime}, 1^{\prime \prime}$-dioxide, $(\mathrm{T} 5 \mathrm{oCx})$ at different relative concentrations (molecular structures in the inset of figure 1, $\mathrm{R}=n$-hexyl, $\mathrm{R}_{1}=$ cyclo-hexyl) in toluene solutions. The diamine derivative, TPD ( $E_{\mathrm{g}} \cong 3.1 \mathrm{eV}$ ) [11], commonly used as a hole-injecting layer in OLEDs, has already been used in binary blends, forming exciplexes with several blueemitting molecules [9]. T5oCx $\left(E_{\mathrm{g}} \cong 2.3 \mathrm{eV}\right)$ was chosen as the representative compound of a wide class of substituted oligothiophenes that were found to be particularly suitable candidates for photonics applications because of their high photoluminescence (PL) efficiency (up to $70 \%$ in the solidstate) [12] and wide colour tunability [4] (both related to their easy functionalization pattern) [13], good chemical stability [14], high solubility in common organic solvents and very good film-forming capability, rendering them excellently spincoating compatible. In this study, $\mathrm{T} 5 \mathrm{oCx}$ was chosen as the red-emitting substituted oligothiophene giving the best EL performances [15].

A calcium electrode followed by an aluminium cap was finally deposited by thermal evaporation $\left(10^{-6} \mathrm{mbar}\right)$. The device characterizations were performed at room temperature in air atmosphere. PL characterization was performed on films deposited onto quartz glass substrates under a He-Cd laser $(\lambda=325 \mathrm{~nm})$ excitation.

The TPD emission spectrum, $F_{\mathrm{H}}$, and T5oCx absorption, $\sigma_{\mathrm{D}}$, are peaked at $400 \mathrm{~nm}$ and $450 \mathrm{~nm}$, respectively (figure 1).

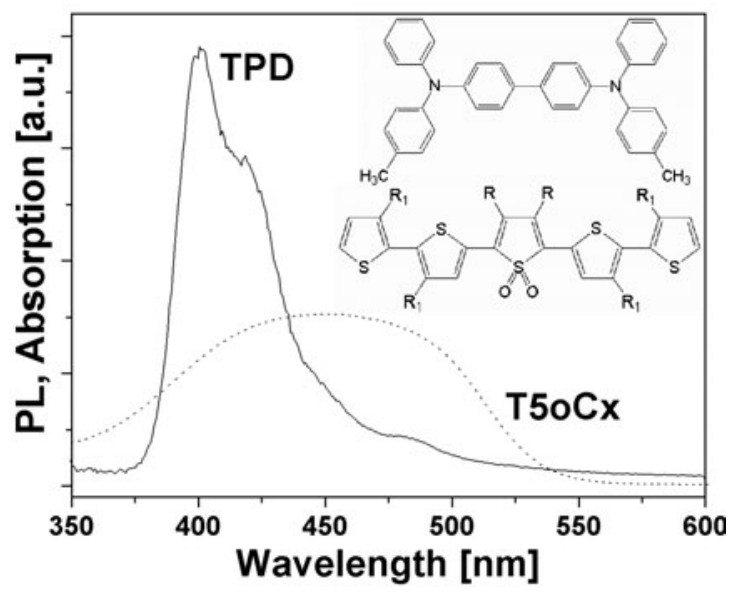

Figure 1. Normalized PL of TPD (- ${ }^{-}$) and absorption spectrum of T5oCx $(\cdots \cdots)$. Inset: chemical structures of the two molecules, TPD (top) and T5oCx (bottom). $\mathrm{R}=\left(\mathrm{CH}_{2}\right)_{5} \mathrm{CH}_{3}$ (n-hexyl), $\mathrm{R}_{1}=\mathrm{C}_{6} \mathrm{H}_{10}$ (cyclo-hexyl).
A strong spectral superposition between $F_{\mathrm{H}}$ and $\sigma_{\mathrm{D}}$ occurs, determining an efficient Förster energy transfer towards the oligothiophene. In solid films an acceptor molecule interacts with a distribution of donors in such a way that the rate of Förster energy transfer $(K)$ via dipole-dipole interaction between two molecules results [16]:

$$
K=4 \pi \int_{r=2 a}^{r=+\infty} \frac{1}{\tau_{\mathrm{H}}}\left(\frac{R_{0}}{r}\right)^{6} \rho \chi r^{2} \mathrm{~d} r=\frac{1}{\tau_{\mathrm{H}}} \frac{R_{0}^{6} \chi}{8 a^{6}},
$$

where $a$ is the radius of the host molecule, $\rho=3 /\left(4 \pi a^{3}\right)$, $\tau_{\mathrm{H}}$ indicates the characteristic time of spontaneous emission from the host material, $R_{0}$ is the Förster characteristic radius, mainly determined by the spectral overlap between $F_{\mathrm{H}}$ and $\sigma_{\mathrm{D}}$ [17], and $\chi$ is the relative molar concentration of acceptors and donors (T5oCx : TPD). The probability, $P$, of exciton transfer from the donor to the acceptor is consequently given by [17]:

$$
P=\frac{K}{\left(1 / \tau_{\mathrm{H}}\right)+K} .
$$

The energy transfer between different molecular species by non-radiative processes like Förster transfer has been exploited as the working principle for multi-layer OLEDs fabricated by high-vacuum thermal deposition [18] and lasing devices $[19,20]$. As shown by equation (1), the efficiency of such processes can be modulated by varying the ratio $\chi=\mathrm{T} 5 \mathrm{oCx}$ : TPD, resulting in effective tuning of the spectral emission. This approach can be a general route to tuning colour from blends of low-molar-mass compounds, allowing achieving balanced white emission without a need for polymeric diluting matrices.

In figure 2(a) we show the PL spectra of T5oCx : TPD blends with relative concentrations (T50Cx : TPD, expressed in number of molecules) between $1: 1$ and $1: 10^{4}$. Concentrations down to $1: 10^{2}$ result in almost complete energy transfer to $\mathrm{T} 5 \mathrm{oCx}$, while for $\chi<10^{-2}$ the blue peak from TPD becomes more and more evident. We emphasize that the lowering of the low-energy peak with decreasing $\chi$ is not linearly proportional to the number of $\mathrm{T} 5 \mathrm{oCx}$ molecules. This indicates that it is not simply a consequence of the reduction of number of the red-emitting species, but originates primarily from the tuning of the Förster rate (equation (1)). In fact, in the absence of energy transfer mechanisms one would expect a linear $\chi$-dependence of the ratio between the emitted intensities from T5oCx and TPD. This would result in the dotted curve in the inset of figure $2(a)^{5}$. In contrast, $P$, which is obtained from the PL spectra by taking into account the luminescence efficiencies of the two species, $\eta_{\mathrm{PL}}^{\mathrm{T50Cx}}=0.22$ [12] and $\eta_{\mathrm{PL}}^{\mathrm{TPD}}=0.35$ [21], exhibits a different behaviour and rapidly tends to unity as $\chi$ increases (inset in figure $2(a)$ ), indicating the presence of effective Förster transfer. In order to have a deeper insight into the efficiency of the exciton transfer from donor to acceptor, we evaluated the Förster radius by correlating it to the transfer probability, $P$, through equations (1) and (2):

$$
R_{0}^{6}=\frac{8 a^{6} P}{\chi(1-P)} .
$$

\footnotetext{
5 The dotted curves in the insets of figure 2 were calculated by the $(P, \chi)$ behaviour expected if the lowering of the low-energy peak was linear with respect to the decrease of the $\mathrm{T} 5 \mathrm{oCx}$ concentration.
} 
(a)

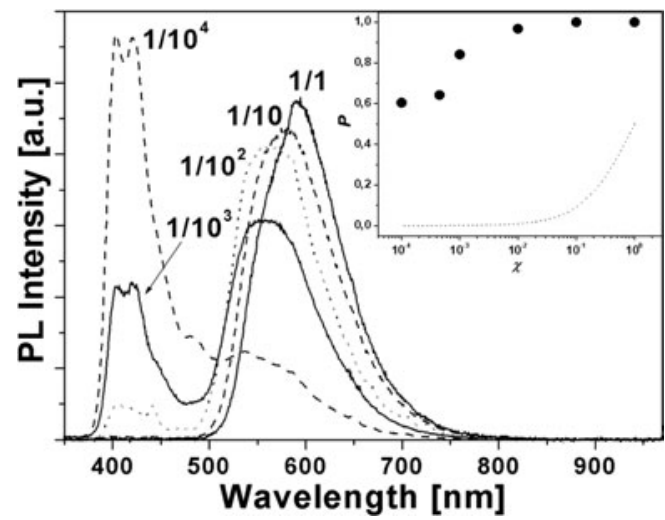

(b)

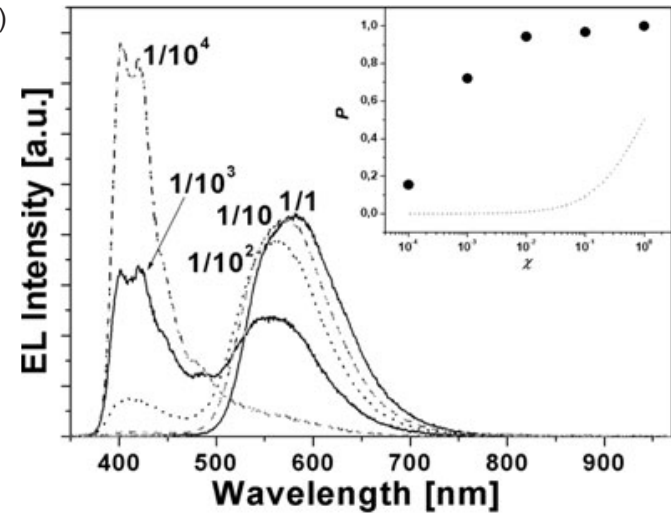

Figure 2. (a) PL spectra of the T5oCx : TPD spin-coated films for different relative concentration, $\chi$. Inset: the dots indicate the donor-acceptor exciton transfer probability, $P$, vs $\chi$, calculated from PL data (see the text). The dotted curve indicates the values of $P$ obtained assuming a linear dependence of the ratio between the emitted intensities from T50Cx and TPD on $\chi$. (b) EL spectra of the OLED device ITO/PEDOT-PSS/TPD-T5oCx/Ca and corresponding transfer probability, $P$, for different $\chi$. Inset: same as for figure $2(a)$.

Taking the radius of an equivalent spherical molecule of TPD to be $a \cong 8.7 \AA$ [21], we obtain $R_{0}$ larger than $50 \AA$. This value is larger than that of a typical blend, DCM2: $\operatorname{Alq}_{3}\left(R_{0}=39 \AA\right)$ [17], which confirms the T5oCx:TPD is a system where Förster transfer is quite effective.

The EL spectra from different T5oCx : TPD concentrations, reported in figure $2(b)$, exhibit the same behaviour as the PL spectra, although the rise of the high-energy peak as $\chi$ decreases is more favoured with respect to PL. This reflects an apparent decrease in exciton transfer probability in EL (inset in figure $2(b)$ ), which can be attributed to the formation of new absorbing species under electrical excitation, as shown for DCM2: $\mathrm{Alq}_{3}$ blends [22]. We underline that, different from previously reported experiments $[1-3,7,8,16,18]$, our OLEDs are based on a single active layer and do not employ dye molecules. The lack of effects of PL quenching induced in dye compounds by the concentration allowed us to effectively investigate the dependence of the Förster exciton transfer probability on the relative concentration of the elements of the blend.

The emitted colour of both PL and EL was characterized, according to the standards of the Commission Internationale de l'Eclairage (CIE), as a point in a two-dimensional space of coordinates, $(X, Y)$ (figure 3). Moreover, by interpolating the dependence of the CIE coordinates on $\chi$ (figure 3),

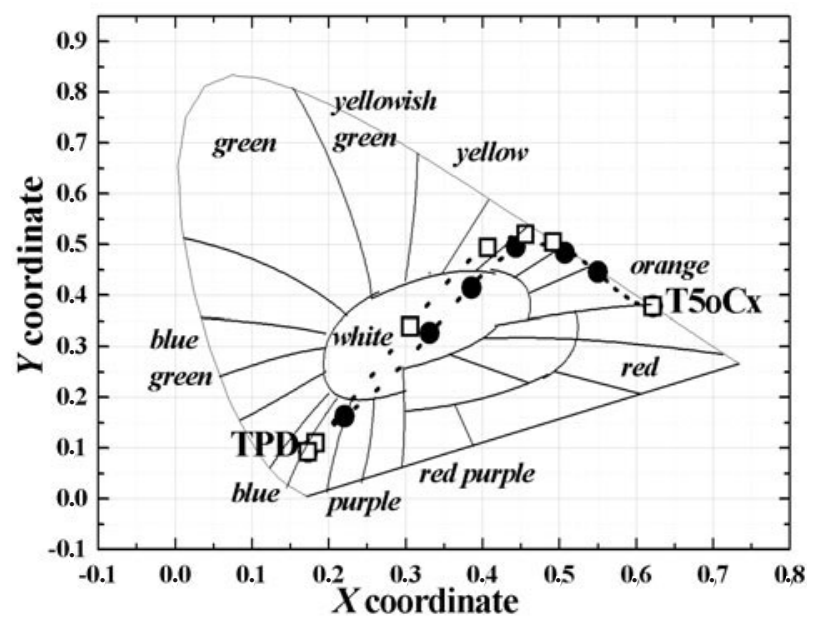

Figure 3. Behaviour with $\chi$ of the CIE coordinates of the PL ( $\bullet$ from right to left: $\chi=1,10^{-1}, 10^{-2}, 10^{-3}, 4.5 \times 10^{-4}, 10^{-4}$ ) and EL ( $\square$, from right to left: pure T5oCx, $\chi=1,10^{-1}, 10^{-2}, 10^{-3}$, $10^{-4}$, pure TPD) spectra. The dotted lines are guides for the eye.

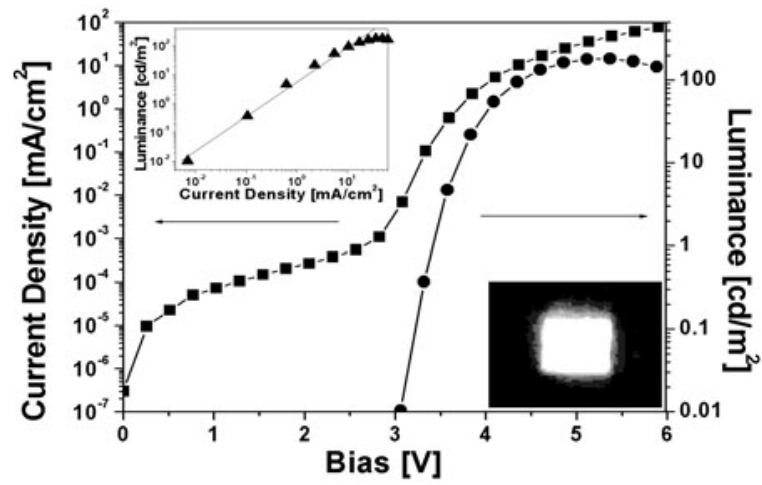

Figure 4. Current density ( $\boldsymbol{\square}$, left-hand scale) and luminance $(\bullet$, right-hand scale) vs applied voltage for the white-emitting OLED $\left(\chi=10^{-3}\right)$. Insets: (top) dependence of the luminance above the threshold on the current density and (bottom) photograph of a typical white OLED at $5 \mathrm{~V}$. The line in the top inset is a linear fit to the experimental data, having a slope coefficient $\cong 1.1$, which indicates an almost linear dependence of the luminance on the current.

it was possible to determine the T50Cx : TPD concentration $\left(4.5: 10^{4}\right)$, resulting in a perfectly balanced white PL emission $(0.33,0.33)$. In EL, it was similarly possible to reach the balanced white region $(0.31,0.34)$ of the CIE diagram with $\chi=10^{-3}$ (inset in figure 4). Moreover, different from other results on Förster transfer-based OLEDs [10], for our devices almost no dependence on the applied voltage was observed in the emitted colour, whose CIE coordinates did not exhibit significant variations on increasing bias. By this route it may be possible to achieve an exact colour coordinate, including balanced white, by binary blends of organic compounds.

Finally, in figure 4 we report the current density and the luminance vs the applied voltage of a typical white single-active-layer T5oCx : TPD OLED. It exhibits a turn-on voltage, $V_{\mathrm{ON}}$, for luminance of $0.01 \mathrm{~cd} \mathrm{~m}^{-2}$, of $3.1 \mathrm{~V}$ and a luminance up to $182 \mathrm{~cd} \mathrm{~m}^{-2}$ (EL efficiency $\sim 5 \times 10^{-1} \mathrm{~cd} \mathrm{~A}^{-1}$ ). All the other devices, spanning from red to blue across the CIE diagram, show comparable values of turn-on bias 
$\left(2 \mathrm{~V} \leqslant \mathrm{~V}_{\mathrm{ON}} \leqslant 3.5 \mathrm{~V}\right)$ and luminance (up to $\left.400 \mathrm{~cd} \mathrm{~m}^{-2}\right)$. The area of our devices was about $2 \mathrm{~mm}^{2}$. We recall that previous studies demonstrated a degradation of OLED performance as the emitting area increases, due to the presence of local defects causing short-circuits and to the high resistivity of the transparent electrode [23]. Further studies are currently in progress in our laboratory to assess the effect of increasing electroluminescent region on device performance, aiming to apply this approach to large-area illuminating OLEDs.

In summary, we reported on an approach to obtaining colour tunability, including white, from binary blends employed as the emitting medium of single-active-layer OLEDs. The method is based on the proper fine control of the efficiency of the non-radiative energy transfer from the high-energy to the low-energy emitter, allowing achievement an exact CIE coordinate by varying the relative concentration of the two molecules of the blends. We successfully experimented this route on the blend T5oCx : TPD. This allows us to cover the CIE diagram, including the balanced white region, with good EL performances, opening up the way to realizing multicolour and white OLEDs by organic binary blends with one-step spin-coating fabrication.

\section{References}

[1] Jordan R H, Dodabalapur A, Strukelj M and Miller T M 1996 Appl. Phys. Lett. 68 1192-4

[2] Xie Z, Feng J, Huang J, Liu S, Wang Y and Shen J 2000 Synth. Met. 108 81-4

[3] Zugang L and Nazare H 2000 Synth. Met. 111 47-51

[4] Anni M, Gigli G, Paladini V, Cingolani R, Barbarella G, Favaretto L, Sotgiu G and Zambianchi M 2000 Appl. Phys. Lett. 77 2458-60

[5] Granström M and Inganäs O 1996 Appl. Phys. Lett. 68 147-9
[6] Tasch S, List E J W, Ekstrom O, Graupner W, Leising G, Schlichting P, Rohr U, Geerts Y, Scherf U and Mullen K 1997 Appl. Phys. Lett. 71 2883-5

[7] Kido J, Shionoya H and Nagai K 1995 Appl. Phys. Lett. 67 2281-3

[8] Higgins R W T, Monkman A P, Nothofer H-G and Scherf U 2001 Appl. Phys. Lett. 79 857-9

[9] Thompson J, Blyth R I R, Mazzeo M, Anni M, Gigli G and Cingolani R 2001 Appl. Phys. Lett. 79 560-2

[10] Cheon K O and Shinar J 2002 Appl. Phys. Lett. 81 1738-40

[11] Burrows P E and Forrest S R 1994 Appl. Phys. Lett. 64 2285-7

[12] Gigli G, Barbarella G, Favaretto L, Cacialli F and Cingolani R 1999 Appl. Phys. Lett. 75 439-41

[13] Barbarella G, Favaretto L, Sotgiu G, Zambianchi M, Bongini A, Arbizzani C, Mastragostino M, Anni M, Gigli G and Cingolani R $2000 \mathrm{~J}$. Am. Chem. Soc. 122 11971-8

[14] Barbarella G, Favaretto L, Zambianchi M, Pudova O, Arbizzani C, Bongini A, Mastragostino M 1998 Adv. Mater. $10551-4$

[15] Gigli G, Inganäs O, Anni M, De Vittorio M, Cingolani R, Barbarella G and Favaretto L 2001 Appl. Phys. Lett. 78 1493-5

[16] Bulovic V, Shoustikov A, Baldo M A, Bose E, Kozlov V G, Thompson M E and Forrest S R 1998 Chem. Phys. Lett. 287 455-60

[17] Bulovic V, Baldo M A and Forrest S R 2001 Organic Electronic Materials ed R Farchioni and G Grosso (Berlin: Springer) pp 402-22

[18] Deshpande R S, Bulovic V and Forrest S R 1999 Appl. Phys. Lett. 75 888-90

[19] Kozlov V G, Bulovic V, Burrows P E and Forrest S R 1997 Nature 389 362-4

[20] Berggren M, Dodabalapur A and Slusher R E 1997 Appl. Phys. Lett. 71 2230-32

[21] Mattoussi H, Murata H, Merrit C D, Iizumi Y, Kido J and Kafafi Z H 1999 J. Appl. Phys. 86 2642-50

[22] Kozlov V G, Burrows P E, Parthasarathy G and Forrest S R 1999 Appl. Phys. Lett. 74 1057-9

[23] Duggal A R, Foust D F, Nealon W F and Heller C M 2003 Appl. Phys. Lett. 82 2580-2 